Atıf için / For Citation: M. YILMAZ, S. F. ÖZMEN, "Kültür Minekop Balığının (Umbrina cirrosa Linnaeus, 1758) Radyolojik Risk Değerlendirmesi”, Süleyman Demirel Üniversitesi Fen Edebiyat Fakültesi Fen Dergisi, 14(2), 269-275, 2019.

\title{
Kültür Minekop Balığının (Umbrina cirrosa Linnaeus, 1758) Radyolojik Risk Değerlendirmesi
}

\author{
Mesut YILMAZ ${ }^{1}$, Süleyman Fatih ÖZMEN ${ }^{2 *}$ \\ ${ }^{l}$ Akdeniz Üniversitesi Su Ürünleri Fakültesi, Su Ürünleri Yetiştiriciliği Bölümü, 07058 Antalya-Türkiye \\ ${ }^{2}$ Akdeniz Üniversitesi Teknik Bilimler MYO, Elektrik ve Enerji Bölümü, 07058 Antalya-Türkiye \\ *yazışılan yazar e-posta: fatihozmen@akdeniz.edu.tr
}

(Alınış / Received: 16.07.2019, Kabul / Accepted: 17.09.2019, Yayımlanma / Published: 30.11.2019)

Özet: $\mathrm{Bu}$ çalışmada Muğla bölgesinde yetiştirilen minekop balığının kas dokusunda doğal radyonüklit $\left({ }^{226} \mathrm{Ra},{ }^{232} \mathrm{Th}\right.$ ve $\left.{ }^{40} \mathrm{~K}\right)$ konsantrasyonları ölçülerek radyolojik risk değerlendirmesi yapılmıştır. Bu amaçla yetiştiriciliğin yapıldığı çiftlikten porsiyonluk balıklar hasat sonrası alınarak kas dokuları ayrılmıştır. Numunelerin yüksek çözünürlüklü germanyum dedektörü ile gama spektrometrik ölçümleri gerçekleştirilmiş ve radyolojik risk faktörleri hesaplanmıştır. Çiftlikte yetiştirilen minekop balıklarının yenilebilen kısımlarında ${ }^{226} \mathrm{Ra}$ aktivitesinin 0,63 ile $4,60 \mathrm{~Bq} \mathrm{~kg}^{-1},{ }^{232} \mathrm{Th}$ aktivitesinin $0,29-4,14 \mathrm{~Bq} \mathrm{~kg}^{-1}$ ve ${ }^{40} \mathrm{~K}$ aktivitesinin $133,19-160,57 \mathrm{~Bq} \mathrm{~kg}^{-1}$ aralığında değiştiği gözlenmiştir. Örneklerin radyum eşdeğer aktivitesi ve yıllık etkin doz ortalamaları sırasıyla $13,79 \pm 0,62 \mathrm{~Bq} \mathrm{~kg}^{-1}$ ve $4,96 \pm 0,07 \mu \mathrm{Sv} \mathrm{y}^{-1}$ olarak hesaplanmıştır. Elde edilen bulgular kültür koşullarında yetiştirilen minekop balıkları için referans oluşturması bakımından önemlidir.

Anahtar kelimeler: Minekop, Su ürünleri yetiştiriciliği, Doğal radyoaktivite, HPGe, Umbrina cirrosa

\section{Radiologic Risk Assessment of Farmed Shi Drum (Umbrina cirrosa Linnaeus, 1758)}

Abstract: In this study, natural radionuclide $\left({ }^{226} \mathrm{Ra},{ }^{232} \mathrm{Th}\right.$ and $\left.{ }^{40} \mathrm{~K}\right)$ concentrations were measured in edible part of shi drum grown in Muğla region and radiological risk assessment was performed. For this purpose, pan size fish were taken from the farm soon after harvesting and fillets were separated. Gamma spectrometric measurements of samples were performed with high resolution germanium detector and radiological risk factors were calculated. It was observed that the activities ranged from 0.63 to $4.60 \mathrm{~Bq}$ $\mathrm{kg}^{-1}$ for ${ }^{226} \mathrm{Ra}$, from 0.29 to $4.14 \mathrm{~Bq} \mathrm{~kg}^{-1}$ for ${ }^{232} \mathrm{Th}$ and from 133.19 to $160.57 \mathrm{~Bq} \mathrm{~kg}^{-1}$ for ${ }^{40} \mathrm{~K}$ in edible parts of farmed shi drum. Radium equivalent activity and annual effective dose averages of the samples were calculated as $13,79 \pm 0,62 \mathrm{~Bq} \mathrm{~kg}^{-1}$ and 4,96 \pm $0,07 \mu \mathrm{Sv} \mathrm{y}^{-1}$, respectively. The findings are important in terms of forming database for shi drum grown in culture conditions.

Key words: Shi drum, aquaculture, natural radioactivity, HPGe, Umbrina cirrosa 


\section{Giriș}

Akdeniz havzası deniz balıkları yetiştiricilik faaliyetleri özellikle çipura ve levrek balıkları (Sparus aurata ve Dicentrarchus labrax) üzerinde yoğunlaşmaktadır. Bu iki türün yoğun üretimi nedeniyle fiyatlarının düşmesi balık üreticilerini yeni tür arayışına itmektedir [1]. Ticari değerinin yüksek oluşu, kültür koşullarına uyumu ve yüksek performansı [2-5] nedeniyle minekop balığ 1 alternatif bir tür olarak üretilmeye başlanmıştır.

Global su ürünleri üretimi 2017 yılında yaklaşık 112 milyon ton olarak gerçekleşmiştir. Türkiye'de ise aynı yıl su ürünleri yetiştiriciliği yoluyla 273.477 ton üretim yapılmıştır. Doğal dağılımı şekil 1'de verilen minekop balığının kültür koşullarında üretimi sadece Akdeniz havzasında Yunanistan, İtalya ve Türkiye' de yapılmakta olup üretim miktarı yıllara göre değişkenlik göstermektedir (Tablo 1). Akdeniz havzasında maksimum üretim 2011 yılında 1057 ton olarak gerçekleşirken, ülkemizde minekop üretimi 2017 y1lında 125 tona yükselmişstir [6].

Tablo 1. Akdeniz havzasında yıllara göre minekop üretim miktarları (Ton) (FAO, 2019)

\begin{tabular}{lcccccccccc}
\hline \multirow{2}{*}{ Ülke } & \multicolumn{10}{c}{ Y1l } \\
\cline { 2 - 12 } & 2008 & 2009 & 2010 & 2011 & 2012 & 2013 & 2014 & 2015 & 2016 & 2017 \\
\hline Türkiye & 0 & 0 & 0 & 0 & 0 & 0 & 39 & 61 & 20 & 125 \\
Yunanistan & 0 & 0 & 0 & 936 & 525 & 308 & 462 & 476 & 128 & 158 \\
İtalya & 45 & 46 & 131 & 121 & 44 & 76 & 45 & 45 & 150 & 150 \\
\hline
\end{tabular}

Ortamdan bulunan terrestriyal radyonüklitler $\left({ }^{238} \mathrm{U},{ }^{232} \mathrm{Th}\right.$ ve $\left.{ }^{40} \mathrm{~K}\right)$ organizma tarafindan çeşitli yollarla alınır ve bünyelerinde biriktirilirler [7]. Besin zinciri yoluyla da bir üst trofik düzeye aktarılırlar [8]. Böylelikle insana kadar ulaşabilecek olan radyoaktif elementlerin kültürü yapılan balıklarda izlenmesi tüketici sağlığının korunması ve üretim kalitesinin yükseltilmesi bakımından önem arz etmektedir.

Literatürde yayın balığı [9] ve gökkuşağı alabalığı [10] gibi yaygın olarak yetiştiriciliği yapılan türlerinin radyonüklit konsantrasyonları ve radyolojik risk değerlendirmeleri üzerine çalışmalar mevcuttur. Ancak minekop balığı hakkında herhangi bir veriye rastlanmamıştır. Bu nedenle Ege Bölgesinde faaliyet gösteren çiftliklerde yetiştirilen minekop balıklarının yenilebilir kısımlarındaki radyonüklit yükleri araştırılmış ve radyolojik risk değerlendirmesi yapılmıştır.

\section{Materyal ve Metot}

\section{1. Örnekleme ve numunelerin hazırlanmast}

Ege bölgesinde faaliyet gösteren üretim çiftliğinden porsiyonluk balık hasatı sonrasında rasgele olacak şekilde ortalama $37,2(36,3-37,7) \mathrm{cm}$ boy ve $568,2(480,6-687,4) \mathrm{g}$ kütleye sahip 20 birey ölü olarak alınmıştır. Tedarik edilen numuneler ağzı sızdırmaz PE torbalara konulup etiketlenerek Akdeniz Üniversitesi $\mathrm{Su}$ Ürünleri Fakültesi Yetiştiricilik Laboratuarına buzla soğutulmuş kutuda taşınmıştır. 


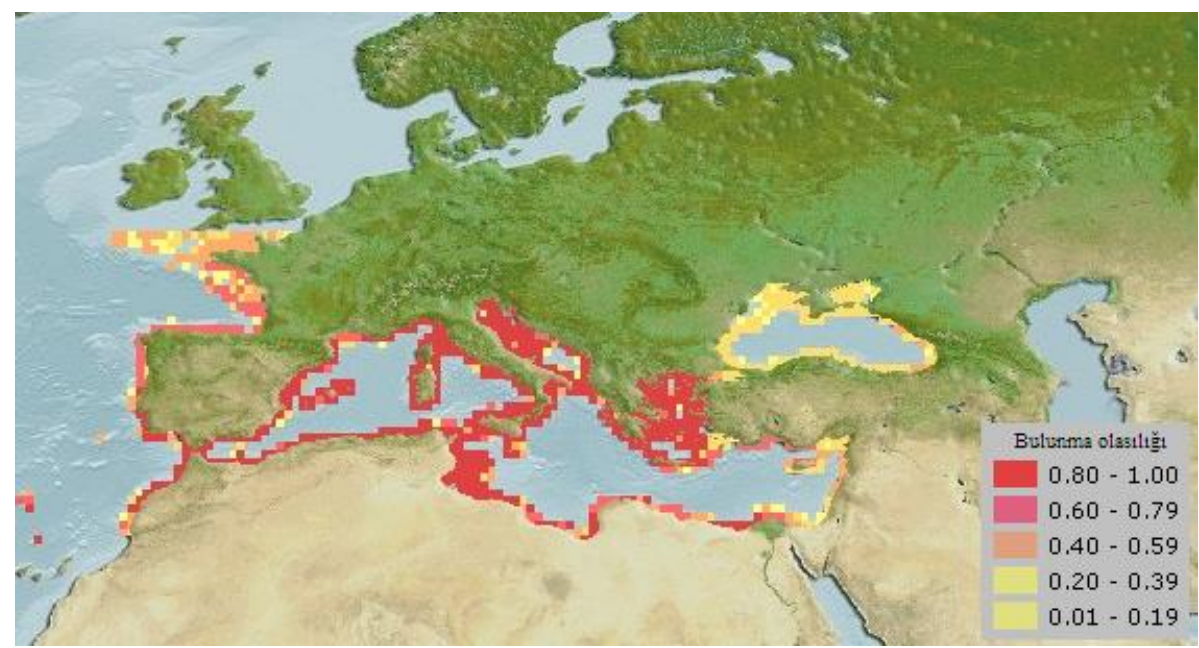

Şekil 1. Minekopun doğal dağılımı

Balık numuneleri laboratuvarda distile su ile yıkanmış ve boy-kütle ölçümleri yapılarak kayda geçirilmiştir. Derili filetoları çıkartılıp kıyıldıktan sonra örnekler ayrı ayrı 150 cc'lik silindirik kaplara doldurulmuştur. Hazırlanan numuneler tartılarak etiketlenmiştir. Örneklerin ağızları gaz sızdırmaz parafilm ile sıkıca kapatılarak ${ }^{226} \mathrm{Ra}-{ }^{222} \mathrm{Rn}$ arasında radyoaktif dengenin oluşması ve Compton bölgesinin kararlı hale gelmesi için $(7 \times 3,86$ gün) yaklaşık 30 gün süreyle $-20^{\circ} \mathrm{C}$ de bekletilmiştir [12].

\subsection{Radyonüklit analizi}

HPGe gama spektrometre sistemi: Çalış1lan örneklerin gamma spektroskopik ölçümleri A.Ü. Fen Fakültesi Fizik Bölümü Gama Spektroskopi Laboratuarında bulunan p-tipi, koaksiyel, elektrik soğutmall, bağıl verimi $\% 40$ ve ${ }^{57} \mathrm{Co}$ için $122 \mathrm{keV}$ 'de $768 \mathrm{eV}$ FWHM ve ${ }^{60} \mathrm{Co}$ için $1332 \mathrm{keV}$ 'de $1.85 \mathrm{keV}$ FWHM değerlerine sahip AMETEKORTEC, GEM40P4 model yüksek saflıkta Germanyum dedektörü ile MAESTRO32 yazılımı kullanılarak gerçekleştirilmiştir. Çalışmada kullanılan HPGe gama spektrometre sisteminin enerji kalibrasyonu, enerjileri $47-1836 \mathrm{keV}$ arasında değişen radyoizotoplar içeren, örneklerle aynı geometrideki karma kaynak ile yapılmıştır [13].

Spektrum analizi: Art ortam (boş numune kabı ile) ve numuneler aynı koşullar altında $86400 \mathrm{~s}$ boyunca sayılmıștır. ${ }^{226} \mathrm{Ra}$ aktivite konsantrasyonları ${ }^{214} \mathrm{~Pb} ; 352 \mathrm{keV}$ ve ${ }^{214} \mathrm{Bi}$; $609 \mathrm{keV}$ enerjili pikler, ${ }^{232} \mathrm{Th}$ aktivite konsantrasyonlar1 ${ }^{228} \mathrm{Ac} ; 911 \mathrm{keV}$ ile ${ }^{208} \mathrm{Tl} ; 583 \mathrm{ve}$ $2615 \mathrm{keV}$ enerjili pikler, ${ }^{40} \mathrm{~K}$ aktivite konsantrasyonları ise $1461 \mathrm{keV}$ enerjili pik kullanılılarak belirlenmiştir. Radyonüklit aktivite konsantrasyonları Denklem 1 kullanılarak tayin edilmiştir.

$$
A=\frac{N / t}{\varepsilon \cdot I_{\mathrm{\gamma}} \cdot m}
$$

Burada A; ilgili radyonüklitin aktivitesi $\left(\mathrm{Bq} \mathrm{kg}^{-1}\right), \mathrm{N}$; ilgili enerjideki toplam net sayım, t; sayım süresi (saniye), $\varepsilon$; HPGe dedektörünün ilgili gama enerjisindeki verimi, I gama ışınının bolluğu ve m; örnek kütlesidir.

\subsection{Radyolojik risk parametreleri}

Toplumun balık tüketiminden dolayı maruz kalacağı radyolojik riski değerlendirmek için; ${ }^{238} \mathrm{U},{ }^{232} \mathrm{Th}$ ve ${ }^{40} \mathrm{~K}$ radyonüklitleriinin spesifik aktivitelerinden kaynaklanan 
radyasyona maruz kalmayı standartlaştırmak için radyum eşdeğer aktivitesi $\left(\mathrm{Ra}_{\mathrm{eş}}\right)$ (Denklem 2) [14], beslenme yoluyla vücuda alınan radyonüklitler ile ilişkili radyasyona bağlı sağlık etkilerinin tahmini için yıllık etkin doz (YED) (Denklem 3) ile Radon ve bozunum ürünlerinden kaynaklanan içsel maruz kalma miktarı iç zarar indeksi $\left(\mathrm{H}_{\text {in }}\right)$ (Denklem 4) [15] parametreleri hesaplanmıştır. Radyolojik risk parametrelerinin hesaplanmasında aşağıdaki formüller kullanılmıştır.

$$
\begin{gathered}
R a_{e s}=C_{U}+1,43 C_{T h}+0,07 C_{K} \\
Y E D=\sum_{i}\left(D_{i} C_{i}\right) * U \\
H_{i n}=C_{U} / 185+C_{T h} / 259+C_{K} / 4810
\end{gathered}
$$

Formüllerde yer alan $\mathrm{C}_{\mathrm{i}}$ ilgili radyonüklitin $\mathrm{Bq} \mathrm{kg}^{-1}$ olarak spesifik aktivitesi, $\mathrm{D}_{\mathrm{i}}$ ilgili radyonüklitin doz dönüşüm katsayısı $\left(\mathrm{Sv} \mathrm{Bq}^{-1}\right)$ ve $\mathrm{U}$ ise balığın yıllık kişi başı tüketim miktarın $\left(\mathrm{kg} \mathrm{y}^{-1}\right)$ temsil etmektedir.

\section{4. Ístatistik analizi}

Çalışmada elde edilen verilerin aritmetik ortalaması ve standart hatası, minimum ve maksimum değerleri, \%25, \%50 ve \%75 dağılım değerlerini içeren tanımlayıcı istatistik analizleri SPSS 22 yazılımı kullanılarak yapılmıştır.

\section{Bulgular}

Sunulan çalışmada Ege Bölgesi'nde yer alan üretim çiftliğinden hasat sonrası alınan minekop balıklarının HPGe gama spektrometre sistemi ile ölçülen ${ }^{226} \mathrm{Ra},{ }^{232} \mathrm{Th}$ ve ${ }^{40} \mathrm{~K}$ radyonüklit aktivite konsantrasyonlarına ilişkin tanımlayıcı istatistik Tablo 2'de verilmiştir. Öte yandan, Türkiye'de ve yurt dışında değişik bölgelerde yapılan çalışmalar da karşılaştırılmak üzere Tablo 3 'te özetlenmiştir.

Tablo 2. Minekop balığı yenilebilir kısımlarının ${ }^{226} \mathrm{Ra},{ }^{232} \mathrm{Th},{ }^{40} \mathrm{~K}\left(\mathrm{~Bq} \mathrm{~kg}^{-1}\right.$ yaş ağırlık) aktivite konsantrasyonlarına ilişkin tanımlayıcı istatistikler

\begin{tabular}{lcccc}
\hline & & ${ }^{226} \mathrm{Ra}$ & ${ }^{232} \mathrm{Th}$ & ${ }^{40} \mathrm{~K}$ \\
\hline $\mathrm{N}$ & Geçerli & 6 & 19 & 20 \\
Ortalama \pm Std. Hata & ÖSA & 14 & 1 & 0 \\
Minimum & & $1.96 \pm 0.59$ & $1,48 \pm 0,31$ & $145,36 \pm 2,13$ \\
Maksimum & & 0.63 & 0,29 & 133,19 \\
& & 4.60 & 4,14 & 160,57 \\
Yüzdelik dilimler & 50 & 0,81 & 0,53 & 137,27 \\
& 75 & 1,64 & 0,91 & 141,64 \\
& & 2,96 & 2,64 & 157,24 \\
\hline
\end{tabular}

ÖSA: Ölçüm sınırının altında, N: numune sayısı

Minekop balığ 1 örneklerinin yenilebilir kısımlarında ${ }^{226} \mathrm{Ra}$ aktivitesinin $0,63-4,60 \mathrm{~Bq}$ $\mathrm{kg}^{-1}$ arasinda, ${ }^{232} \mathrm{Th}$ aktivitesinin $0,29-4,14 \mathrm{~Bq} \mathrm{~kg}^{-1}$ arasinda ve ${ }^{40} \mathrm{~K}$ aktivitesinin 133,19 - 160,57 arasında değiştiği tespit edilmiştir.

Organizmada gözlemlenen radyonüklit konsantrasyonları besin ve içinde yaşadığı ortamdan etkilenmektedir [7]. Bu bağlamda Üretim kalitesinin arttırılabilmesi için minekop balıklarının yenilebilir kisımlarında tespit edilen ${ }^{226} \mathrm{Ra},{ }^{232} \mathrm{Th}$ ve ${ }^{40} \mathrm{~K}$ aktivitesinin kaynaklarının tespiti yönünde ayrıntılı çalışmalara ihtiyaç vardır. 
Tablo 3. Türkiye'de ve diğer ülkelerde yapılan çalışmalar $\left(\mathrm{Bq} \mathrm{kg}^{-1}\right)$

\begin{tabular}{lcccc}
\hline Tür & ${ }^{226} \mathrm{Ra}$ & ${ }^{232} \mathrm{Th}$ & ${ }^{40} \mathrm{~K}$ & Kaynak \\
\hline Yayın Balı̆̆1 & 0,41 & 0,89 & 99 & {$[9]$} \\
Tırsi & 4,5 & 11,0 & 37,4 & {$[16]$} \\
Barbun & 2,66 & 1,26 & 120 & {$[17]$} \\
Hint sazanı & 0,9 & 1,0 & 110 & {$[18]$} \\
Ot sazanı & 1,2 & 0,8 & 83 & {$[18]$} \\
Inci kefali & 0,57 & 0,022 & 319 & {$[19]$} \\
Gökkuşağ1 alabalığ1 & 0,76 & 1,03 & 101,72 & {$[10]$} \\
Mavi yüzgeçli orkinos & 0,26 & 0,42 & 96,30 & {$[10]$} \\
Minekop & $1,96 \pm 0,59$ & $1,48 \pm 0,31$ & $145,36 \pm 2,13$ & Bu çalışma \\
\hline
\end{tabular}

Minekop balığı üzerine yapılmış herhangi bir çalışmaya rastlanmadığından, literatürde yer alan diğer balıkların içerdiği radyonüklit konsantrasyonları ile kıyaslanmıştır. Tablo 3'e göre farklı türlerde yapılan çalışmalarda ${ }^{226} \mathrm{Ra}$ aktivite konsantrasyonlarının 0,26-4,5 $\mathrm{Bq} \mathrm{kg}{ }^{-1},{ }^{232} \mathrm{Th}$ aktivite konsantrasyonlarının 0,42-11,0 $\mathrm{Bq} \mathrm{kg}^{-1}$ ve ${ }^{40} \mathrm{~K}$ aktivite konsantrasyonlarının ise 37,4-319 Bq kg-1 aralığında değiştiği görülmektedir. Mevcut çalışmada tespit edilen minekop balığı yenilebilir kısımlarına ait ${ }^{226} \mathrm{Ra},{ }^{232} \mathrm{Th}$ ve ${ }^{40} \mathrm{~K}$ aktivite konsantrasyonlarının Türkiye'de ve diğer ülkelerde yapılan çalışmaların sonuçlarıyla uyum içerisinde olduğu gözlenmektedir.

Çalışma kapsamında incelenen balık örneklerinin ${ }^{226} \mathrm{Ra},{ }^{232} \mathrm{Th}$ ve ${ }^{40} \mathrm{~K}$ aktiviteleri kullanılarak hesaplanan [Radyum eşdeğer aktivite $\left(\mathrm{Ra}_{\mathrm{es}}\right)$, İç zarar indeksi $\left(H_{\text {in }}\right)$ ve Yıllık etkin doz (YED)] radyolojik risk parametreleri Tablo 4'te listelenmiştir.

Tablo 4. Minekop balıkları yenilebilir kısımlarının radyolojik risk parametrelerine ilişkin tanımlayıcı istatistikler

\begin{tabular}{lcccc}
\hline & & $\mathrm{Ra}_{\mathrm{eS}}\left(\mathrm{Bq} \mathrm{kg}^{-1}\right)$ & $\mathrm{H}_{\text {in }}$ & YED $\left(\mu \mathrm{Sv} \mathrm{y}^{-1}\right)$ \\
\hline $\mathrm{N}$ & Geçerli & 20 & 20 & 20 \\
Ortalama \pm Std. Hata & ÖSA & 0 & 0 & 0 \\
Minimum & & $13,79 \pm 0,62$ & $0,04 \pm 0,00$ & $4,96 \pm 0,07$ \\
Maksimum & & 10,97 & 0,03 & 4,54 \\
& 25 & 21,14 & 0,07 & 5,48 \\
Yüzdelik dilimler & 50 & 11,84 & 0,03 & 4,68 \\
& 75 & 12,78 & 0,03 & 4,83 \\
Dünya ort. [20] & 15,53 & 0,05 & 5,36 \\
\hline \multicolumn{2}{c}{ ÖSA: Ölçüm sinırının altında, N: numune sayis1 } & 370 & $\leq 1$ & $200-800$ (ortalama 290) \\
\hline
\end{tabular}

Minekop balığı yenilebilir kısımları radyum eşdeğer aktivitesi $10,97-21,14 \mathrm{~Bq} \mathrm{~kg}^{-1}$ aralığında değişmekte olup ortalama $13,79 \pm 0,62 \mathrm{~Bq} \mathrm{~kg}^{-1}$ olarak hesaplanmıştır. Benzer şekilde [21] dönüşüm faktörleri ve Balıkçılık ve Su Ürünleri Genel Müdürlüğü [22] tarafından yayınlanan kişi başı yıllık su ürünleri tüketimi $(5,5 \mathrm{~kg})$ değerleri kullanılarak hesaplanan örneklerin yıllık eşdeğer aktivitelerinin ortalama 4,96 $\pm 0,07 \mu \mathrm{Sv} \mathrm{y}^{-1}$ olup, $4,54-5,48 \mu \mathrm{Sv} \mathrm{y}^{-1}$ aralığında değiştiği görülmüştür. Radyum eşdeğer aktivitesinin ve yıllık etkin doz değerlerinin UNSCEAR 2000'de önerilen $\left(\mathrm{Ra}_{\mathrm{es}} 370 \mathrm{~Bq} \mathrm{~kg}{ }^{-1}\right.$ ve YED

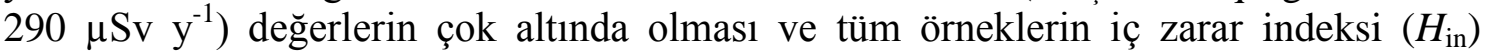
değerlerinin 1'den küçük olması kültür minekop balığının tüketilmesinin radyolojik açıdan insan sağlığı için olumsuz bir etki yaratmayacağını göstermektedir.

\section{Sonuç ve Yorum}

Sonuç olarak, çiftlik koşullarında yetiştirilen minekop balıklarının yenilebilir kısımlarında belirlenen ${ }^{226} \mathrm{Ra},{ }^{232} \mathrm{Th}$ ve ${ }^{40} \mathrm{~K}$ aktivite konsantrasyon değerleri bu alanda literatürdeki eksikliği tamamlamakta ve yetiştiriciliği yapılan diğer türlerle kıyaslanabilir nitelikte bir veri seti oluşturmaktadır. Doğal yayılım alanı Akdeniz havzası olan ve sadece İtalya, Yunanistan ve Türkiye'de alternatif tür olarak yetiştiriciliği yapılan minekop balıklarının yenilebilir 
kısımlarında gözlenen aktivite konsantrasyonlarının ve hesaplanan radyolojik risk parametrelerinin tüketici sağlığı bakımından sorun teşkil etmeyecek seviyelerde olduğu ortaya konmuştur.

Ayrıca, balık dokularında tespit edilen doğal radyonüklitlerin yetiştiricilik ortamındaki kaynaklarının (yem, su, ilaç, havuz veya kafes materyalleri) tespiti ve üretim kalitesini yükseltecek yöntemlerin araştırılacağı çalışmalara ihtiyaç duyulmaktadır.

\section{Teşekkür}

Yazarlar olarak, numunelerin gama spektrometrik ölçümleri için araştırma laboratuarını tahsis eden Akdeniz Üniversitesi Fizik Bölümü Öğretim Üyesi Prof. Dr. İsmail BOZTOSUN'a yardım ve desteklerinden dolayı teşekkürü borç biliriz.

$\mathrm{Bu}$ araştırma, kamu, ticari ve/veya kar amacı gütmeyen sektörlerdeki finansman kuruluşlarından herhangi bir destek almamıştır.

Sunulan çalışmada kullanılan balıklar hasat sonrası ölü olarak örneklendiğinden herhangi bir etik prosedür izlenmemiştir.

\section{Kaynakça}

[1] B. Basurco, E. Abellan, "Finfish species diversification in the context of Mediterranean marine fish farming development," in Options Méditerranéennes., No. 24. Marine Finfish Species Diversification; Current Situation and Prospects in Mediterranean Aquaculture. E. Abellan, B. Basurco (eds.). C.I.H.E.A.M., Zaragoza, Spain 1999, pp. 9-25.

[2] G. Fabi, L. Fiorentini, "Catch and growth of Umbrina cirrosa (L.) around artificial reefs in the Adriatic Sea," Boll. Oceanol. Teor. Appl., 11, 235-242, 1993.

[3] P. Cardellini, A. Franceson, S. Zanella, G. Bozzato, P. Benedetti, N. Borgoni, A. Barbaro, "Captive rearing of shi drum, Umbrina cirrosa (L.), in different thermal conditions," Biol. Mar. Mediterr., 6, 287-290, 1999.

[4] C.C. Mylonas, G. Georgiou, D. Stephanou, T. Atack, A. Afonso, Y. Zohar, "Preliminary data on the reproductive biology and hatchery production of the shi drum (Umbrina cirrosa) in Cyprus," in Cahiers Options Méditerranéennes, Mediterranean Marine Aquaculture Finfish Species Diversification, vol. 47, B. Basurco (Ed.). C.I.H.E.A.M., Zaragoza, Spain, 2000, pp. 303-312.

[5] A. Barbaro, A. Franceson, D. Bertotto, G. Bozzato, I. Di Maria, P. Patarnello, F. Furlan, L. Colombo, "More effective induction of spawning with long-acting GnRH agonist in the shi drum, Umbrina cirrosa L. (Sciaenidae, Teleostei), a valuable candidate for Mediterranean mariculture," J. Appl. Ichthyol., 18, 192-199, 2002.

[6] FAO (2019, 16 Temmuz). Fisheries and aquaculture software. FishStatJ - Software for Fishery and Aquaculture Statistical Time Series. In: FAO Fisheries and Aquaculture Department [online]. Available: http://www.fao.org/fishery/statistics/software/fishstatj/en

[7] E.V.D. Stricht, R. Kirchmann, “ Radioecology, Radioactivity \& Ecosystems," Fortemps, Liège.

[8] IAEA (International Atomic Energy Agency), "Sediment distribution coefficients and concentration factors for biota in the marine organisms," IAEA Technical report series, No. 422, Vienna, 2004.

[9] J. Billa, F. Han, S. Didla, H. Yu, J. Dimpah, O. Brempong, S. Adzanu, "Radioactivity studies on farm raised and wild catfish produced in Mississippi, USA," J. Radioanal. Nucl. Chem., 307 (1), 203-210, 2016.

[10] F.K. Görür, R. Keser, N. Akçay, S. Dizman, "Radioactivity and heavy metal concentrations of some commercial fish species consumed in the Black Sea Region of Turkey," Chemosphere, 87, 356-61, 2012.

[11] Fishbase (2019, 16 Temmuz). Computer generated distribution maps for Umbrina cirrosa (Shi drum), with modelled year 2100 native range map based on IPCC A2 emissions scenario. www.aquamaps.org, version of Aug. 2016. [Online]. Available: https://www.aquamaps.org/receive.php?type_of_map=regular\#.

[12] G. Yaprak, M.A.A. Aslani, "External dose-rates for natural gamma emitters in soils from an agricultural land in West Anatolia," J. Radioanal. Nucl. Chem., 283, 279-287, 2010. 
[13] S.F. Ozmen, A.Cesur, I. Boztosun, M. Yavuz, "Distribution of natural and anthropogenic radionuclides in beach sand samples from Mediterranean Coast of Turkey," Radiat. Phys. Chem., 103, 37-44, 2014.

[14] J. Beretka, P.J. Mathew, "Natural radioactivity of Australian building materials, industrial wastes and by-products," Health Phys., 48 (1), 48-87, 1985.

[15] R. Krieger, " Radioactivity of construction materials," Betonw Fertigtl Technol, 47, 468-473, 1985.

[16] B.B. Bolaji, , D.S. Francis, H. Ibitoruh, "Human health impact of natural and artificial radioactivity levels in the sediments and fish of Bonny estuary, Niger Delta, Nigeria," Challenges, 6, 244-257, 2015.

[17] C.C. Goddard, C.P. Mathews, J. Al Mamry, "Baseline radionuclide concentrations in Omani Fish," Mar. Pollut. Bull., 46, 914-917, 2003.

[18] S. Tahir, A. Alaamer, M. Ayub, M. Khan, "Radiometric analysis of samples of domestic fish species and radiological implications," Health Phys., 98(5), 741-744, 2010.

[19] S. Erenturk, S. Yusan, D.A. Turkozu, Z. Camtakan, M.K. Olgen, M.A.A. Aslani, S. Aytas, M.A. Isik, "Spatial distribution and risk assessment of radioactivity and heavy metal levels of sediment, surface water and fish samples from Lake Van, Turkey," J. Radioanal. Nucl. Chem., 300 (3), 919-931, 2014.

[20] UNSCEAR "Sources and effects of ionizing radiation," Report to General Assembly, with Scientific Annexes, United Nations Scientific Committee on the Effect of Atomic Radiation, 2000.

[21] ICRP "Compendium of Dose Coefficients based on ICRP Publication 60," ICRP Publication 119, Annual ICRP 41(Suppl.), 2012.

[22] BSGM (Balıkçılık ve Su Ürünleri Genel Müdürlüğü) (2019, 6 Mayıs). T.C. Tarım ve Orman $\begin{array}{lllll}\text { Bakanlığ su ürünleri } & \text { [Online]. Available: }\end{array}$ https://www.tarimorman.gov.tr/sgb/Belgeler/SagMenuVeriler /BSGM.pdf. 